\title{
Traversées féminines de l'Atlantique noir, dans des œuvres de Fabienne Kanor et de Léonora Miano
}

MARJOLAINE UNTER ECKER

Aix-Marseille Université

凶 marjolaine.unter-ecker@univ-amu.fr

RÉSUMÉ. Fabienne Kanor et Léonora Miano, auteures afro-descendantes d'expression française, explorent dans leurs œuvres I'Atlantique noir (Gilroy, 1993). Celui-ci dépasse les frontières traditionnellement établies, puisque s'y produisent de nombreuses circulations et que les identités et les cultures qui s'y déploient se définissent par l'hybridité ; aussi, on peut s'interroger sur les capacités émancipatrices que cet espace propose pour les catégories de races et de genres. C'est ce que cet article se propose d'étudier, à travers une étude littéraire portant sur un corpus constitué d'un recueil de textes pour la scène, Red in blue trilogie (Miano, 2015) et d'un roman,

MOTS-CLÉS:

Atlantique noir ; déportation transatlantique ; personnages féminins ; résistance ; hybridité Humus (Kanor, 2006). Ceux-ci re-localisent les discours historiques sur l'esclavage, notamment en les ancrant dans les récits des vécus et des expériences intimes de personnages féminins, participant de ce fait du projet de "contre-culture de la modernité " qu'est celui de l'Atlantique noir.

\section{Pour citer cet article}

Unter Ecker, M. (2020). Traversées féminines de l'Atlantique noir, dans des œuvres de Fabienne Kanor et de Léonora Miano. Hybrida, 1, 67-92. http://doi.org/10.7203/ HYBRIDA.1.16878 
RESUMEN. Fabienne Kanor y Léonora Miano autoras francófonas de descendencia africana exploran en sus obras el Atlántico negro (Gilroy, 1993). Ese espacio traspasa las fronteras establecidas tradicionalmente, porque allí ocurren numerosos tránsitos de identidades y culturas definiéndose por el mestizaje. Asimismo, podemos hacernos preguntas sobre las capacidades emancipadoras que este lugar propone para la categoría de raza y género. Es lo que va a estudiar este artículo, mediante un estudio literario relativo a un corpus compuesto por una colección de textos escritos para el teatro, Red in blue trilogie (Miano, 2015) y una novela, Humus (Kanor, 2006). Esos textos reubican la novela histórica de la esclavitud, en particular arraigándose a los relatos de las vivencias y las experiencias íntimas de los personajes femeninos, participando así en el proyecto de "contra-cultura de la modernidad" que es el del Atlántico negro.

ABSTRACT. Fabienne Kanor and Léonora Miano, who are French-speaking African-descendant writers, explore the Black Atlantic (Gilroy, 1993) in their works. Because of the movements that go through it, identities and cultures that unfold there are defined by hybridity. Thus, Black Atlantic goes beyond traditional established borders. That's why we could also ask ourselves about the emancipatory possibilities that this space offers for races and genders. This is what this article aims to study, through a literary study that will focus on a novel, Humus (Kanor, 2006), and a collection of three plays, Red in blue trilogie (Miano, 2015). These texts re-localize historical discourse on slavery, particularly by telling it through female characters' intimate experiences. Thus, these stories take part in the Black Atlantic project of "counterculture of modernity".
PALABRAS CLAVES:

Atlántico negro; deportación transatlántica; figuras femeninas; resistencia; mestizaje

KEY-WORDS:

Black Atlantic; literature; gender studies; slavery; hybridity 
Atlantique noir ", notion forgée par Paul Gilroy en 1993, représente un vaste lieu de culture qu'a en partage la diaspora africaine et qui est né à travers la traite transatlantique. Dans cet espace circulent des individus, des idées et des pratiques artistiques, entremêlant les influences africaines, américaines et européennes. Pour signifier le caractère composite de ces cultures, Paul Gilroy emploie l'image d'un navire négrier qui circule entre les trois continents. A partir de l'exploration de cet espace, il envisage l'Atlantique noir comme un espace de « contre-culture de la modernité »(Gilroy, 2017, p. 30). Dans la conception hégémonique, les cultures sont délimitées par des frontières nationales et raciales; dans la conception diasporique, et donc celle de l'Atlantique noir, elles vont au-delà de celles-ci, et se caractérisent par des fluctuations.

C'est aussi à travers cet aspect mouvant que l'auteure franco-camerounaise Léonora Miano détermine son identité :

Je la dis frontalière, ancrée, non pas dans un lieu de rupture, mais, au contraire, dans un espace d'accolement permanent. La frontière, telle que je la définis et l'habite, est l'endroit où les monde se touchent, inlassablement. C'est le lieu de l'oscillation constante : d'un espace à l'autre, d'une sensibilité à l'autre, d'une vision du monde à l'autre. [...] La frontière évoque la relation. Elle dit que les peuples se sont rencontrés, quelquefois dans la violence, la haine, le mépris, et qu’en dépit de cela, ils ont enfanté du sens. (Miano, 2012, p. 25)

En considérant la frontière à partir du sens subsaharien ancien, Miano l'appréhende comme « une espace de médiation » (Miano, 2016, p. 46), et se l'approprie pour signifier sa manière d'habiter le monde. Elle lui permet notamment de représenter le contact entre ses appartenances multiples, qui sont à la fois africaines et occidentales. Née au Cameroun en 1973 et résidant aujourd'hui en France, elle se définit comme une «Afro-occidentale parfaitement assumée » (Miano, 2012, p. 26), affirmant par ailleurs que de toute façon, « être un Africain, de nos jours, c'est être un hybride culturel » (Miano, 2012, p. 28). Selon l'auteure, du fait de l'histoire coloniale, les cultures africaines contemporaines sont imprégnées des traces de la présence occidentale, ce qu'elle ne perçoit pas comme une acculturation inéluctable, mais au contraire comme une possibilité de « pouvoir choisir le meilleur de chaque culture ( Miano, 2012, p. 28). En outre, lorsqu'elle évoque son adolescence, Miano souligne également l'influence importante de la culture afro-américaine. La lecture d'auteurs des Amériques et des Caraibes, comme Baldwin et Césaire (Miano, 2012, p. 13) a constitué pour elle une révélation, en ce qu'ils lui ont fait prendre conscience de sa condition noire. Sa production littéraire, qui comprend principalement des romans, mais aussi des essais et 
des textes pour la scène, reflète son hybridité culturelle. Elle rassemble les trois pôles que rapproche l'Atlantique noir, à la fois à travers son esthétique et les sujets explorés : elle écrit en français, « mais les références, les images qu'elle déploie sur la page, appartiennent à d'autres sphères » (Miano, 2012, p. 29) ; la structure de ses textes s'inspire des musiques afro-américaines, et notamment du jazz; enfin, ses œuvres mettent en scène des personnages afro-descendants, afropéens ${ }^{1}$ ou subsahariens, et leurs négociations identitaires en prise avec le continent d'origine, que ce soit dans le contexte de l'esclavage (La Saison de l'ombre, 2013), de l'Afrique postcoloniale, contemporaine (L'intérieur de la nuit, 2005 ; Contours du jour qui vient, 2006 ; les Aubes écarlates, 2009 ; Crépuscule du Tourment tome 1, 2016 et tome 2, 2017) et future (Rouge impératrice, 2019) ou encore dans celui de la "post-migration" (Afropean Soul et autres nouvelles, 2008 ; Tels des astres éteints, 2008b ; Blues pour Élise, 2010 ; Écrits pour la parole, 2012).

Le parcours et les œuvres de Fabienne Kanor, romancière, journaliste et cinéaste s'inscrivent eux aussi dans le triangle transatlantique qui rassemble l'Afrique de sa diaspora :

Dans mes livres, la plupart de mes personnages sont noirs. Cela veut dire qu'ils ont des histoires communes, qu'elles se passent en Afrique, dans la Caraïbe, ou dans l'Amérique noire. Je me sens profondément de ce terroir noir, que je porte comme une couleur politique. (Kanor, 2014)

« Ce terroir noir » constitue un espace de « résolution » des appartenances de cette auteure, qui souligne son sentiment de «déterritorialisation ». À travers cette notion, que Deleuze et Guattari ont conceptualisée pour signifier des processus de décontextualisation, elle manifeste son sentiment d'être « à côté de soi-même » (Kanor, 2014), du fait de son absence d'ancrage dans les territoires auxquels son parcours la rattache. Née en 1970 en France métropolitaine, elle témoigne de l'exclusion et du racisme qu'elle y a subis. Elle se sent aussi éloignée et étrangère de la Martinique, île d'origine de ses parents, où elle n'a pas grandi ${ }^{2}$. À la recherche d'un lieu à soi, elle ne cesse depuis de parcourir le monde : elle a vécu en Angleterre, au Sénégal, au Cameroun, a voyagé en Afrique de l'Ouest et en Amérique du Sud, a circulé au sein des Caraibes et réside aujourd'hui aux États-Unis, espaces visités et vécus dans lesquels elle puise pour créer.

1 Miano emploie ce néologisme pour définir les Afrodescendant·e·s d'Europe.

2 Voir à ce propos: Gladys M. Francis. (2016). Fabienne Kanor «l'Anté-Llaise Par Excellence»: Sexualité, Corporalité, Diaspora et Créolité. The French Forum, 41(3), 273-278 (pp. 274-275). https://doi. org/10.1353/frf.2016.0035 
En effet, les parcours de ses personnages quadrillent de manière multidirectionnelle et multi-temporelle l'espace atlantique : de l'Afrique vers les Amériques dans le contexte de la Déportation transatlantique (Humus, 2006) ; des Amériques caribéennes vers l'Europe dans le cadre des migrations organisées par le BUMIDOM dans les années 1960 (Jambe Dlo, 2008) et le récit de personnages afro-caribéens installés en métropole (D'eaux douces, 2004); d'Amérique du Sud vers l'Europe dans un roman qui met en scène des personnages brésiliens qui se réfugient en France (Les chiens ne font pas des chats, 2008) ; d'Afrique vers l'Europe, notamment dans le contexte des migrations contemporaines (Faire l'aventure, 2014b) ; d'Europe vers les Afriques ou d'Europe vers l'Amérique du Nord à travers les voyages de certains de ses personnages (Anticorps, 2010 ; Je ne suis pas un homme qui pleure, 2016 ; Louisiane, 2020).

La démarche littéraire de Fabienne Kanor tout comme celle de Léonora Miano s'apparente à une volonté d'inclure les expériences afro-descendantes au sein d'une mémoire prétendument universelle, mais qui reste pourtant conditionnée par la domination occidentale. Or, c'est aussi en cela que consiste le projet de « contre-culture de la modernité » que Paul Gilroy définit dans son ouvrage. Il s'agit pour lui de déconstruire la modernité, qui est traditionnellement pensée comme «blanche et homogène » et qui a été influencée par « l'euro-ethnocentrisme de la pensée des Lumières » (Cellier, 2018, p. 180). Pour cela, le chercheur remet notamment en question les rapports de domination à l'œuvre dans le projet colonial et esclavagiste, à l'instar de ceux que le maître entretient avec l'esclave. Il s'agit dès lors de déconstruire cette "relation caractéristique de la modernité »(Gilroy, 2017, p. 98), en adoptant désormais le point de vue de celles et ceux qui en furent victimes. C'est aussi ce que revendique Miano, qui défend par exemple la notion de "Déportation transatlantique des Subsahariens » (Miano, 2016, p. 141-183) face au termes usuels, comme "traite négrière », " commerce triangulaire » etc., jugeant que ceux-ci sont limités au point de vue de ceux qui en ont bénéficié, et sont impropres à rendre compte de la complexité humaine qu'a instituée l'esclavage, dont il faudrait rendre compte dans sa globalité : des vécus liés à la capture, jusqu'à ceux des plantations, en passant par la traversée transatlantique et l'enfermement dans la cale des navires négriers.

La littérature s'impose alors comme un support idéal pour ce projet : la stylistique des textes, la mise en intrigue, la construction des figures narratives etc., inclut une approche sensible des expériences et de l'histoire. En permettant aux lecteur·rice·s de s'immerger jusque dans les questionnements intimes de personnages et notamment de ceux dont les présences au monde sont peu représentées, elle permet de décloisonner les frontières entre soi et l'altérité : 
En tant quécrivain, on ouvre des portes. On ne sait pas toujours où l'on va. Mais au bout du compte on a envie de raconter une histoire, de faire parler des gens qui parfois n'ont pas du tout la parole [...] Comme écrivain, nous avons envie de sentir les émotions et de les restituer. En tant que romancière, c'est aussi important pour moi de retrouver des arrière-pays, et de les habiter. (Kanor, 2014)

C'est donc en explorant ces « espaces autres ", comme ceux qu'habite la diaspora afro-descendante, que la littérature participe d'un projet de déconstruction de l'hégémonie. Elle permet alors de déplacer les perspectives sur les identités et les cultures, qui dans le cadre de l'Atlantique noir, tout comme dans la pensée de la frontière quétablit Miano, se caractérisent par des dynamiques de transformation constantes et invitent à « l'inversion du rapport entre la marge et le centre qui est au cœur des discours magistraux de la race maîtresse »(Gilroy, 2017, p. 98). Or, cette reconfiguration de la hiérarchie qu'impose la norme peut s'appliquer à diverses " classifications » sociales. On peut alors se demander dans quelles mesures elle pourrait aussi s'attacher aux questions de genres, d'autant plus que dans le contexte de l'Atlantique noir, celles-ci croisent la race et la classe : ainsi, les conditions des Subsahariennes déportées, femmes, noires et esclavagisées, se caractérisent par « l'intersectionnalité »(Crenschaw, 2005). C'est ce à propos de quoi notre étude littéraire, qui portera sur un roman de Fabienne Kanor, $\mathrm{Hu}$ mus (2006) ainsi que sur un recueil de textes pour la scène de Léonora Miano intitulé Red in blue trilogie (2015), se propose d'apporter des pistes de réflexion.

\section{Fragmentations en Atlantique noir}

\subsection{Lecture genrée de la désagrégation du « monde connu »}

Dans son texte "Parole due », Miano propose des outils pour relocaliser la tragédie atlantique dans les expériences des déporté•e•s. Or, selon elle, «pour les victimes du crime, les transportés / déportés et leurs proches, il n'y eut pas de traite, mais une déflagration, la désagrégation du monde connu » (Miano, 2016, p. 160), ce dont rend compte le roman Humus de Kanor, qui raconte le parcours de femmes subsahariennes devenues esclaves. Les différents chapitres débutent généralement par une description synesthésique et bucolique du territoire d'origine en période précoloniale. Celui-ci est figuré par l'équilibre entre la nature, les éléments et les sociétés humaines, incluant aussi certaines fois une harmonie entre les genres, hommes et femmes y vivant ensemble paisiblement :

Dans ce pays suspendu comme un songe, j'entends descendre et grossir le fleuve. Prendre de force la terre, les hommes, les bêtes. Rien ne lui résiste. Dans le ciel, des 
oiseaux passent. [...] En bas, les enfants visent, hurlent de joie quand la proie tombe. Sous le soleil généreux, je regarde les gosses faire jusqu'à ce qu'une voix les rappelle à lordre. Les cases se ferment. [...]

Au pays, les hommes ignorent ce que pleurer veut dire, leur langue ne connait pas ce mot. Elle en a d'autres, plein : manger, danser, cuire, chasser, pêcher, cultiver.

Au pays, les femmes sont toujours gaies. Quoi qu'elles fassent, mettent une chanson dans leur bouche qu'elles sucent à longueur de temps comme une friandise. (Kanor, 2006, p. 18)

En ces débuts de récit, les personnages féminins sont racontés à travers le rôle phare qu'elles jouent au sein des communautés : fille, mère, grand-mère, " ancienne ", pilier du foyer, reine, guerrière, etc., elles se caractérisent alors par leur agentivité : elles sont celles qui « font » le jour (Kanor, 2006, p. 31), celles qui savent « où poser les pieds » (Kanor, 2006, p. 32), celles qui « ouvrent la marche » (Kanor, 2006, p. 82).

Mais rapidement, les textes changent de registre : en racontant la « violence fondatrice » (Etoke, 2010,p. 30) qu'a constituée la Déportation transatlantique pour celles qui en furent victimes, ils laissent place à une tension tragique et s'orientent vers une suspension de la dynamique des existences. C'est ce que figure le décor de la pièce "Révélation » (Miano, 2015, pp. 11-51) : « Le sol est d'anthracite. Il n’y a aucune végétation. Le cri d'un oiseau se fait entendre. Ce n'est pas un chant. C'est un cri, incontestablement » (Miano, 2015, p. 17). Lunivers mis en scène représente une forme de chaos, qui est dû au refus des " âmes à naître » à se réincarner dans un monde où la mémoire de l'esclavage n'est pas résolue. Aussi, les esprits des collaborateur'rice's subsaharien'ne's à la traite sont convoqué·e·s pour expliquer leurs actes, au sein de ce qui s'apparente à un procès. Leurs justifications témoignent de la fracture que l'esclavage a engendrée au sein des communautés (village, ethnie etc.), incluant celles qui sont liées au genre. Lorsque Ofiri, personnage féminin de la pièce, a été confrontée à la stérilité, elle a en effet capturé et vendu des femmes de son propre royaume, alors même qu’elle avait été élue " prêtresse » et " gardienne » de la présence d'Inyi-figure féminine du divin-auprès des siennes :

OFIRI. [...] Oui, j'ai usé de mon statut pour livrer des femmes aux hommes venus de l'autre côté. Celles dont la grossesse débutait à peine. Parfois, elles ignoraient encore tout de leur état. À la faveur d'assemblées nocturnes qui se tenaient dans un sanctuaire, je les faisais disparaître. Vêtues de blanc, nous nous rendions en procession au bord de l'océan. Il s'agissait d'accomplir un rituel. Une fois arrivées, je leur donnais à boire une potion sédative. Elles s'endormaient vite. On ne les embarquait pas aussitôt. Les étrangers les voulaient conscientes. Ils devaient s'assurer de leur bon état de santé. Elles s'éveillaient à l'aube, nues, pieds et poings liés, au fond d'une cabane proche de là. J'agissais pour me venger. Apaiser ma douleur... [...] J'entends encore les cris de ces femmes. Les malédictions qu'elles ont proférées. Certaines mont traitée de sorcière. (Miano, 2015, p. 46) 
Ses agissements vont à l'encontre des principes de solidarité à travers lesquels peuvent être représentées les sociétés matriarcales et matrilinéaires de l'Afrique précoloniale. Selon l'historien Cheick Anta Diop, c'est justement l'arrivée des Européens qui bouscula " la collaboration et l'épanouissement harmonieux des deux sexes ${ }^{3}$ (Diop, 1979, p. 220) en privant les femmes du rôle central qu'elles y jouaient jusqu'alors et en y instaurant le règne du patriarcat.

De fait, l'arrivée des négriers au sein des territoires d'origine s'illustre par des violences de rapport de genre. Le motif du viol, outil d'asservissement répandu sur les navires négriers et dans les plantations, illustre particulièrement l'instauration d'une domination exacerbée des hommes sur les femmes, ce que souligne par ailleurs l'hyperbole de type numéral employée dans cet extrait :

Une nuit, ils mont mangé le ventre. L'homme était seul mais c'est comme s'ils étaient cent. Je n'avais plus aucune larme quand il est entré. Je songeais seulement à mon doigt dans la gorge. Ce doigt qui ne suffirait jamais à tout enlever. J'ai pensé qu'il faudrait que j'en mette deux, la main, le bras. Jusqu'à ce que toute l'eau de l'homme parte. [...] À présent, j’ai mal quand je regarde ma main. Mes jambes flageolent. Je saigne, serre les poings [...]. (Kanor, 2006, p. 19-20)

Au fur et à mesure de la transportation ${ }^{4}$ puis de la déportation, le corps féminin que s'est approprié le maître se voit dépossédé des capacités agentives qu'il avait dans le «monde connu ». Parallèlement à cela, les repères spatio-temporels des captives se dissolvent. L'une des hérö̈nes du roman Humus se demande alors : «Où sont mes sables, mes fleurs, mes soleils? Mon tout petit pays avec ses ciels qui pleurent et rient ? Cette maison est trop vaste, ce n'est pas la mienne. Pas cela, pas moi, ce corps qui pousse » (Kanor, 2006, p. 59). Arraché à sa terre natale, marchandisé, enfermé, le corps de la femme esclave n'est plus sien. Tout au plus il enferme, mais il ne s'habite plus, ce que figure le texte lorsqu'il l'évoque comme une entité indépendante de la voix narrative : ainsi, la Vieille doit " traîner [s]on corps toute la journée, $l e^{5}$ tenir à distance des coups de fouet »(Kanor, 2006, p. 32). Les fluides corporels, qui font généralement écho à l'énergie de la vie et de la régénération et qui comportent en ce sens une symbolique

3 Voir aussi à ce propos l'ouvrage l'Unité culturelle de l'Afrique noire: domaine du patriarcat et du matriarcat dans l'Antiquité classique (1982).

4 Léonora Miano emprunte ce terme à la chercheure Lucie Nkaké, pour signifier le déplacement des captif·ve·s depuis leur village d'origine jusqu'à la côte africaine (Nkaké, Lucie. 1997. La mémoire de la capture. UNESCO / société africaine de culture).

5 Nous soulignons. 
liée au féminin, ou du moins à la maternité, se dissolvent ici : soit ils se perdent dans ceux de l'homme qui asservit, soit ils sont happés par celle de l'Atlantique, le Passage du milieu figurant pour les déportées une constante négociation de la vie avec la mort :

L'eau m'avait quittée lorsque le bateau toucha terre.

Comme une éponge, la mer avait tout pris, mes larmes, mes sangs, cette eau qui jute pour dire et prendre le plaisir. Une vieille branche, voilà ce qu'elle avait fait de moi la mer. Une ombre aux mamelles muettes dont nul n'aurait su dire si elle avait été femme, homme, chien. (Kanor, 2006, p. 38)

\subsection{La condition des femmes esclaves au prisme des violences intersectionnelles}

Cette dernière phrase est significative de la condition que subissent les femmes noires dans la cale du navire négrier, puis dans les plantations. Tous deux constituent des lieux où la race se construit, puis se cristallise : c'est la croyance en une hiérarchie raciale qui justifiait le projet esclavagiste des Européens. En invoquant leur supériorité sur les peuples d'Afrique, et en représentant ceux-ci sous les traits de leur altérité négative, les Occidentaux ne considéraient pas l'identité et l'humanité des captif•ve•s :

Les Noirs, tels que l'Europe les construisit au cours des siècles de DTS, ne furent que des ombres sans nom, sans visage. Ils furent ce que montrent les nombreuses représentations de corps entassés dans l'entrepont des navires : des êtres sans appartenance aucune, privée d'individualité. (Miano, 2016, pp. 144-145)

L'entreprise d'asservissement, qui va de pair avec l'institution de la race, se décline en effet à travers « la destruction de la dignité humaine, le retrait des noms et des statuts, le démantèlement des groupes afin qu'il n'existe plus de langue commune et la suppression de tout signe manifeste d'un quelconque héritage africain » (hooks, 2015 , p. 61). Toutes ces particularités sont gommées par la couleur de peau et les représentations essentialisantes qui lui sont rattachées. Aussi, quand le personnage de la Reine souhaite faire valoir son statut social auprès du capitaine du navire négrier, elle se heurte à l'indifférence, car celui-ci « dit que tout nègre c'est nègre et qu'il lui importe peu de savoir qui a fait qui, qui était qui. Ici, tous les esclaves se ressemblent. Aucune distinction. Aucun privilège » (Kanor, 2006, p. 181). L'absence de considération envers le statut social du personnage, justifiée ici par la race, témoigne aussi de l'instauration d'une logique de classe, l'une constituant la modalité de l'autre (Gilroy, 2017, p. 158). Dans une perspective marxiste, la classe dominée est celle qui fournit les moyens de production, ce à quoi sont réduits les personnages esclavagisés ; à cette perception 
économique s'ajoute la privation de pouvoir politique, social ${ }^{6}$ et de droits dont les esclaves étaient totalement dépourvus, ainsi que le déterminent par exemple des textes législatifs comme le Code Noir? ${ }^{7}$.

Les rapports de genre entrent aussi en compte dans cette situation où « le Noir est placé en position subordonnée dans un système dualiste qui reproduit la domination de la blancheur, alliée à la rationalité et à la masculinité »(Gilroy, 2017, p. 98). Tout comme la race, le genre apparaît comme une construction négative en miroir du maître - blanc et homme -, ainsi que le signifie l'intertexte à Simone de Beauvoir qui apparaît dans Humus : « L'eau sale, la chienne, la femme. C'est à cause d'eux, de leur chose qui j’en suis sûre me détraque. On ne naît pas femme, on le devient à leur contact. La manière qu'ils ont de nous regarder, de nous toucher, de nous remplir» (Kanor, 2006, p. 104). La violence esclavagiste et les représentations dépréciatives de la race qui y sont liées s'exerçaient en effet d'une manière particulièrement exacerbée sur les femmes, ainsi que l'explique bell hooks ${ }^{8}$ dans son ouvrage Ne suis-je pas une femme?. Dans les navires négriers, où les membres de l'équipage étaient principalement des hommes, la nudité des femmes esclaves les exposait constamment aux agressions sexuelles (hooks, 2015, p. 61) ; dans les plantations, elles travaillaient aussi au sein même des foyers, et cette proximité avec les familles faisait d'elles les premières cibles de leurs maîtres. Aussi, le genre est appréhendé comme un support d'aliénation, qu'il soit porté à son paroxysme par une appréhension uniquement sexuée - dans le cadre du viol, par exemple, où la femme est considérée comme un objet sexuel - ou qu'il soit totalement nié. « Ni homme, ni femme ", dit la Vieille, ce qui ne signifie pas ici une émancipation des rôles traditionnellement dévolus, mais au contraire une accentuation de la déshumanisation de l'esclave féminine, perception qui puise dans la « matrice de la race » :

6 Voir à ce propos les théories de Max Weber.

7 Le Code Noir est un texte officiel, ayant été amendé plusieurs fois, qui légiférait sur le statut des esclaves et le droit que les maîtres avaient sur eux dans les colonies françaises. À propos de l'absence de droit, on peut relever, entre autres, dans la version datant de 1685 l'article 31, qui stipule que «ne pourront aussi les esclaves être partie, ni être en jugement, ni en matière civile, tant en demandant qu'en défendant [...]»; l'article 38: «L'esclave fugitif qui aura été en fuite pendant un mois à compter du jour que son maître l'aura dénoncé en justice, aura les oreilles coupées, et sera marqué d'une fleur de lys sur une épaule, et s'il récidive un autre mois [...] aura le jarret coupé et sera marqué d'une fleur de lys sur l'autre épaule, et la troisième fois, il sera puni de mort»; ou encore l'article 44, qui déclare: «les esclaves êtres meubles et entrer comme tels dans la communauté $[\ldots] »$.

8 De son vrai nom Gloria Jean Watkins, bell hooks a choisi son nom de plume en hommage à sa mère et à sa grand-mère. Nous écrivons son pseudonyme en minuscules, conformément à la pratique de l'auteure, qui considérait les majuscules comme un signe de culte de la personnalité. 
[...] l'indistinction des caractères sexués est un signe d'infériorité et de basse naissance. [...] On retrouve des considérations similaires dans les récits de voyages ou les traités naturalistes qui remarquent constamment combien les peuples dits sauvages ne sont pas ou peu physiologiquement différenciés sexuellement, ce dont témoignent classiquement l'absence de barbe chez les hommes et l'absence ou le peu de règles chez les femmes. Les systèmes racistes du milieu du XIX ${ }^{\mathrm{e}}$ siècle systématiseront de telles affirmations. (Dorlin, 2009, p. 211)

Autrement dit, les catégories genrées s'appliquent aux êtres humains ; or, les esclaves, et par extension les personnages noirs ne sont pas considérés comme tels sous le regard raciste, et ne peuvent donc pas non plus être réellement définis comme homme ou femme. En effet « dire d'un esclave, vivant au début du XIX e siècle, ou d'un Noir du Mississipi dans les années quarante, qu'il est actif, qu'il détient le pouvoir, qu'il est autonome et que la société est à son image, apparaît éminemment problématique ». De même « proclamer que les femmes sont discriminées parce qu'elles sont des femmes relève [...] d'un privilège de femmes blanches, puisque, historiquement, les femmes noires nont pas été à proprement parler considérées comme de «vraies femmes » (Dorlin, 2005, pp. 87-88). Cet argument a poussé des afro-féministes, comme les membres du Combahee River Collective, à préférer se rallier aux luttes d'émancipation liées à leur communauté de couleur et à se désolidariser des mouvements féministes blancs, ceux- ci étant seulement définis sur des critères de genre, excluant les discriminations raciales et classistes.

L'enfermement dans la cale du navire négrier et plus généralement dans la condition d'esclave constitue donc un lieu privilégié pour le récit de leurs oppressions croisées. C'est pourquoi nous les appréhendons comme des espace-temps cristallisant "l'intersectionnalité », que Kimberlé Crenschaw (2005) définit comme l'interaction entre les conditions du genre et de la race. Exclues de la norme que l'on rattache à la représentation de la lutte féministe - que l'on pense principalement comme blanche - et de celle que l'on rattache à la lutte antiraciste - que l'on pense principalement comme masculine - les femmes noires sont alors doublement privées de stratégies de défense. C'est pourquoi elles sont finalement reléguées « en un lieu difficilement accessible au langage » (Crenshaw, 2005, p. 53), qui est aussi celui de la multiplication de la violence qu'elles subissent. C'est ce qu'illustre, par métonymie, le personnage de la Muette, qui avec la capture, perd la faculté de la parole et en oublie jusqu'à son prénom :

Sans bouche pour les nommer, les mots sont tombés. Joie, sourire, enfance, sauterelles, baobabs... Ils ont coulé les mots, sans rien dire. Ce n'est que longtemps après que j'ai su. Quand il n'est plus rien resté, j'ai ouvert la bouche. Le vide. Le silence [...] Dans ma bouche, il est un mot qui refuse de se dire. Pèse comme ces fers qui 
nous enchaînent à la mer. Le jour où ma langue s'est mise à tourner, j'ai prié pour qu'il tombe. Mais il n'est pas venu. Mon doigt était trop court. Ohé ! Quelqu'un a-t-il trouvé mon nom ? Ohé ! C'est celui-là que je cherche. J'en avais un avant d'arriver ici, tous les hommes possèdent cela. Allongée sur le dos, je passe en revue chaque bout de moi. C'est comme un jeu, ma main qui marche sur mon corps, traverse les jambes, les hanches, contourne le ventre. Pas lui! Surtout pas, depuis qu'ils l'ont cassé. Une fois, c'est arrivé. Je veux dire par là que l'homme est entré en moi, a gâté cette musique qui berce l'enfance.

Je hais les hommes! (Kanor, 2006, pp. 19-23)

La dissolution de l'identité du personnage, figurée ici par la perte du nom de naissance ${ }^{9}$ s'établit parallèlement à la dislocation du corps violé et esclavagisé. Dans les textes qui s'ancrent dans l'Atlantique noir, cette violence «sectionne »- mot que l'on retrouve d'ailleurs aussi dans la notion d'intersectionnalité, et que l'on peut interpréter comme significative de la fragmentation du sujet lorsqu'il est confronté à la multiplication des oppressions -, en atteignant, à son paroxysme, des actes de torture, qui s'articulent tant de façon physique que psychologique, comme par exemple dans cet extrait du roman Humus qui raconte la fuite d'un esclave :

Un mois, ils l'ont traqué. Des nuits à secouer l'île dans tous les sens [...]

$\mathrm{Au}$ trente et unième jour, alors que nous coupions la canne, il y eut comme un coup de pétoire dans le ciel. La machette trembla [...] Les regards tous tombèrent sur ces chevaux dévalant la plaine, traînant derrière eux le cadavre d'un garçon.

Ce n'est pas tout. Le maître, fou de colère, exige que la mère vienne identifier le corps. Elle accourt, regarde le Blanc, lui répète que ce n'était pas son enfant, ce prétendu fils [...]. Le maître perd patience. Vingt-cinq coups de fouet. La femme continue de nier. Cinquante. On lui ordonne, puisque ce n'est pas son fils, d'en finir avec le macchabée [...] Dix jours après, elle craquait. Une folie qui, avant de traverser son corps, dansait autour d'elle comme une toupie. [...] Bientôt la mort viendrait. (Kanor, 2006, p. 40)

La « violence du texte » (Gontard, 1981), qui laisse d'ailleurs planer le doute sur l'identité du jeune esclave tué, témoigne ici d'une multiplication des supplices, ceux-ci s'exerçant aussi sur des sentiments humains, comme l'amour maternel qui « est interdit Aux esclaves »(Miano, 2015, p. 85). Cet extrait fait écho à des pratiques de torture employées au temps de l'esclavage, où, des mères étaient forcées d'assister à l'exécution de leur fils qui avaient marronné, ce qu'Elsa Dorlin explique en ces termes :

9 Remarquons que, une fois vendus, les esclaves étaient souvent renommés par leur maître, qui les considérait comme leur propriété. Voir à ce propos: Laurent, A. (1994). Rituel du nom et du nommer dans la culture afro-américaine: les récits d'esclaves, in Parcours identitaires. Presses Sorbonne Nouvelle. http:// books.openedition.org/psn/4701 
« la justice coloniale [...] veut apprendre aux esclaves que le droit de conservation ne leur appartient ni en propre ni à celle qui leur a donné la vie, mais qu'il ne relève que du seul intérêt de leur maître ». (Dorlin, 2017, pp. 26-27)

\section{Les résistances à l'anéantissement}

\subsection{Pouvoirs performatifs des corps...}

Néanmoins, même dans les situations de désarmement les plus totales, les populations en situation de domination ont développé des pratiques d'auto-défense, notamment par le biais de leur corps ${ }^{\mathbf{1 0}}$. En contexte esclavagiste, les femmes qui étaient d'autant plus démunies du fait de leur genre, ont développé des stratégies qui leur étaient propres. Des mères pratiquaient par exemple l'infanticide, qui constituait la seule issue pour épargner à leurs enfants une vie d'asservissement. En ce sens, il est investi par des personnages féminins comme un acte de résistance, et plus globalement - et ce également dans d'autres contextes relevés par Mona Chollet -, comme des « armes de protestation, à la fois contre la condition faite aux femmes et contre l’ordre social en général »(Chollet, 2018, p. 88), ce dernier pouvant être par exemple celui qui est régi par la race :

MARESHA. [...] Comme nombre de nos sœurs de la montagne, comme bien des femmes dans les habitations d'en bas, j'ai utilisé tous les remèdes prescrits par nos mères. Pour ne pas donner des bras neufs à l'esclavage. Quand notre enfant est venu au monde, j'ai pensé à lui ôter la vie. Le supprimer, afin qu'ils ne connaissent jamais le fouet. (Miano, 2015, pp. 79-80)

Dans le contexte de l'Atlantique noir, l'infanticide constitue ainsi un acte de transgression de la domination des Blancs sur les esclaves. En le commettant, les personnages féminins privent leurs maîtres des forces de production à travers lesquelles ils les considèrent. Ainsi, il figure plus globalement une modalité de liberté autre que celle qui prévaut en contexte hégémonique, puisqu'il va à l'encontre des principes à travers lesquels leurs maîtres se proclament. En effet, encore aujourd'hui, l'infanticide constitue en Occident un tabou et il «suscite l'horreur de la société, qui voit en celle qui l'a commis un monstre »(Chollet, 2018, p. 89), ce qui est aussi lié au genre : lorsqu'il est commis par des femmes n'ayant pas choisi de devenir mère, il figure particulièrement

10 Dans son ouvrage Se défendre (2017), Elsa Dorlin propose une analyse de plusieurs de ces pratiques, qui s'articulent dans des contextes spatio-temporels multiples (esclavage, nazisme, ségrégation par exemple). 
un acte de réappropriation de soi. C'est aussi ainsi qu'on pourrait le considérer au temps de l'esclavage, où la fertilité constituait une valeur marchande : pour les maîtres, il était moins couteux de faire « reproduire » les esclaves qu'ils avaient déjà que d'en acheter de nouveaux ${ }^{11}$.

Le motif de l'infanticide s'inscrit plus globalement dans une appréhension de la mort comme transgression du pouvoir hégémonique, et peut ainsi être analysé comme un exemple de contre-discours de la modernité :

Le choix répété de la mort plutôt que de la servitude exprime un principe de négativité qui s'oppose à la logique formelle et au calcul rationnel, caractéristiques de la pensée occidentale moderne, qui s'incarnent, chez Hegel, dans le choix que fait l'esclave de la servitude plutôt que de la mort. (Gilroy, 2017, p. 132)

La mort, lorsqu'elle est décidée plutôt que d'être subie, peut être ainsi lue en contexte oppressif comme une forme d'«éthiques martiales de soi » (Dorlin : 2017) : paradoxalement, elle permet au sujet dominé de se réapproprier sa propre existence et sa «dignité humaine », qui "dans la situation la plus tragique qui soit », "se traduit par des appels à mourir les armes à la main. Se battre, et peut-être survivre, mais avant tout se faire les hérauts de la vie contre la mort »(Dorlin, 2017, p. 67). C'est aussi le discours que tient le personnage de l'Amazone du roman Humus, qui clame « La mort plutôt que l'esclavage !» (Kanor, 2006, p. 87), et qui considère donc la mort comme une alternative à la déshumanisation que l'esclavage et son interaction avec d'autres formes d'oppressions vise à produire. La transcription du saut sous le point de vue de la Muette performe alors une émancipation du corps asservi :

J'ai.

Je.

Nous avons sauté.

Ensemble. Nous avons.

Sauté. Mer. Sautez!

Nous.

L'avons fait. (Kanor, 2006, p. 21)

Si la syntaxe désarticulée et la mise en page de ce passage pourraient illustrer les « expériences de la fragmentation et de l'éclatement [d]'un corps [...] malmené par

11 Voir à ce propos: Biezunska-Malowist, I. \& Malowist, M. (1966). La procréation des esclaves comme source de l'esclavage. Quelques observations sur l'esclavage dans l'Antiquité, au Moyen Age et au cours des temps modernes, in Mélanges. K. Michalowski. https://bon.edu.pl/media/book/pdf/La_procreation_des_esclaves-IBM_i_MM.pdf 
la déportation et l'injure - (Gilroy, 2017, p. 15), il n'en reste pas moins qu'elle place en son centre le « Je » du personnage. Ce «Je » est sujet d'une action - celle du saut. Celui-ci témoigne du potentiel agissant du corps dominé, et reconfigure en ce sens l'espace de soumission qu'il constituait a priori en un outil de libération, ce que permet aussi la force collective que signifie le «nous » : c'est parce que les esclaves sautent en nombre que leur acte est retentissant. Comme l'infanticide, il prive des maîtres de «bras neufs » et constitue en ce sens une tactique de défense face au pouvoir négrier.

De manière plus globale, les corps dominés sont mis en valeur à travers des processus d'écriture qui visibilisent les personnages féminins de l'Atlantique noir, doublement exclus du discours de la modernité du fait de leur race et de leur genre. Leur apparence physique individuelle est par exemple largement soulignée : ainsi, les textes de Red in blue trilogie comptent de nombreuses didascalies initiales qui précisent avec minutie les tenues, les coiffures, les accessoires etc., que les comédiennes devraient porter. La dimension raciale est par contre exclue de ces précisions, et le corps est raconté plutôt que d'être exposé, ce qui résonne de manière spécifique pour les personnages féminins, qui en contexte occidental se heurtent à "l'érotisation exotique fantasmée » et à une « racisation érotique »(Chalaye, 2020, p. 111). L'œuvre fictionnelle s'appréhende comme un espace où l'essentialisation est déconstruite au profit d'une affirmation des pouvoirs performatifs du corps dominé. À travers le motif des fluides évoqués précédemment - «l'eau des larmes, l'eau du sang qui vient ou pas, l'eau de la mer, l'eau du vinaigre qui désinfecte, l'eau des hommes quand ils vous prennent, l'eau des naissances, l'eau des rivières d'autrefois et dont l'on se souvient encore " (Kanor, 2013, p. 971) - le corps féminin déporté se fond alors dans l'Atlantique noir, non plus pour s'y dissoudre, mais au contraire pour se reconfigurer : il devient un espace où se formule un potentiel transformateur de la fragmentation initiale en survie, à savoir la « capacité à être qui subsiste après la perte de points de référence essentiels à l'équilibre de l'individu » (Etoke, 2010, p. 31).

\section{2. ... et des voix}

La littérature qui explore la mémoire de l'esclavage participe de cette survie, puisqu'elle permet de faire revivre des individus dont les parcours n'ont pas été consignés dans la mémoire collective, et de diffuser l'histoire de destins qui se sont joués dans les marges de l'histoire. C'est particulièrement le cas des slaves narratives, récits d'esclaves publiés aux États-Unis dès la fin du XVIII ${ }^{\mathrm{e}}$ siècle, qui visaient à dénoncer le système esclavagiste dans une perspective abolitionniste : parce que «l'irruption de la voix de l'esclave inscrit sa présence sur la scène historique et universelle » (Aurélia, 
2013, pp. 1-2), ces écrits déjouent le schéma hégémonique et excluant que constitue, pour les esclaves, la modernité. Néanmoins, ainsi que le constate Dominique Aurelia, ces textes ont majoritairement été écrits par des hommes. On peut alors supposer que l'intersectionnalité s'inscrit aussi dans le patrimoine littéraire : parce que « la question de genre est un facteur de division interne et [parce que] l'aborder dans les communautés non blanches revient à importer les préoccupations des femmes blanches dans un contexte où elles sont non seulement déplacées, mais aussi néfastes » (Crenshaw, 2005, p. 64), elle n'a pas trouvé sa place dans ces récits-témoignages caractéristiques de la culture afro-américaine, qui visaient avant tout à dénoncer la condition de l'esclave, dont le point de vue masculin constituait la norme.

Des auteures contemporaines de l'Atlantique noir cherchent alors à « re-territorialiser la vision, le langage » (Miano, 2016, p. 143) sur la Déportation transatlantique des Subsaharien·ne·s, en complétant, via la fiction, les absences de l'histoire, et notamment celles qui concernent les expériences féminines. Certes, Humus s'inspire d'une anecdote historique datant de 1774, découverte dans le journal de bord du capitaine du navire négrier, qui évoque le saut de « 14 femmes noires toutes ensemble et dans le même temps, par un seul mouvement...» (Kanor, 2006, p. 11). Pour autant :

Cette histoire n'est pas une histoire, mais une tentative de glissement, là où il n'y a plus de témoins pour dire, là où l'homme, plongé dans l'obscurité des mers, dans ce noir-bleu qui n'en finit pas, affronte la pire épreuve qui soit : la mort de la parole, l'aporie. Comme ces ombres jadis enchaînées, le lecteur est dès lors condamné à ne plus bouger. Juste écouter, sans autres distraction, ce chœur de femmes. Entendre encore, jusqu'au bout, au risque de s'étourdir, ces cœurs battants. (Kanor, 2006, p.14)

Si l'archive historique indique que certaines de ces femmes moururent et que d'autres survécurent, elle ne donne aucune précision sur leur identité, leur parcours ou sur ce qu'elles devinrent par la suite. Kanor choisit alors non pas de continuer l'histoire du point de vue hégémonique - qui correspondrait au point de vue du capitaine du navire, homme, blanc et négrier, dont le récit a de toute façon été conservé à travers le journal de bord que l'on peut aujourd'hui encore consulter à Nantes -, mais de restituer, avec toute la dimension sensible que la démarche artistique inclut, les récits des destins individuels de ces femmes dont les récits nont pas été consignés. La caractéristique féminine des personnages devient alors performative au sein même de l'esthétique des œuvres et des motifs qu'elles incluent, l'écriture devenant elle-même " féminine ", au sens où l'entend Hélène Cixous. Selon cette dernière, l'écriture féminine est en effet déterminée par : la centralité du corps, qu'on a « a honteusement appris [aux femmes] à ignorer, à frapper de la bête pudeur» (Cixous, 1975, p. 55), et qui constitue un lieu commun dans le roman 
Humus, tout comme dans les textes de Miano, qui sont destinés à la scène ; lécriture de l'intime, qui se décline dans notre corpus à travers l'inscription de la petite histoire dans la grande histoire, ainsi que celle de l'inconscient, que figurent l'errance des flux de conscience et les récits de la folie qui jalonnent Humus; enfin, c'est aussi à travers la valorisation de la voix que Cixous caractérise cette écriture, élément qui est une spécificité des slaves narratives et qui détermine aussi la stylistique des œuvres étudiées ici.

En effet, Humus, roman chorale qui a d'ailleurs d'abord été un texte pour la scène $^{12}$, comporte de nombreuses marques d'oralité. La narration choisie est interne et on y trouve des formules d'appel comme "Oyez sœurs de chaîne, jusqu'au bout mon histoire!» (Kanor, 2006, p. 79) ainsi que des apostrophes au lecteur - « je vous le dis » (Kanor, 2006, p. 81). Le roman est construit à partir d'une structure polyphonique, qui fait écho à la structure « antiphonique » de l'Atlantique noir. Gilroy emploie cette notion, qu'il emprunte à la musique, pour faire référence aux « conversations et rencontres qui s'établissent entre le sujet racial fragmenté, incomplet, inachevé, et les autres » (Gilroy, 2017, p. 149) : tout en formant ensemble une œuvre commune - le projet du saut dans le monde du texte, et le roman en tant qu'unité fictionnelle dans le monde du lecteur -, chaque chapitre est consacré à l'une des captives, à son parcours individuel, et témoigne à son échelle de la pluralité des manières d'être femme. L'identité particulière de chacune des esclaves est soulignée par les titres des chapitres, qui renvoient à ce qui les caractérise par rapport aux autres captives. Ces appellations se réfèrent par exemple à la classe sociale : l'une d'entre elle est reine tandis qu'une autre a été son esclave dans son royaume d'origine, l'Amazone faisait partie d'une armée au service d'un roi, l'Employée est au service du capitaine etc. Elles sont de différentes générations : la Vieille est une femme âgée, la Petite est une adolescente, les Jumelles semblent également être relativement jeunes etc. Toutes ne sont pas des héroïnes : plusieurs d'entre elles témoignent de leurs faiblesses et certaines d'entre elles sont considérées comme des « traîtres " par les autres. La Blanche entretient une relation amoureuse et sexuelle avec le Capitaine ; l'Employée reste passive devant la souffrance vécue dans les cales dont elle est témoin : «Bien sûr j'entendais leurs cris, les chaînes qui grincent, ces têtes qui s'acharnaient à battre la coque. Je voyais bien que quelque chose ne tournait pas rond en bas mais qu'aurais-je pu faire? On ne me paie pas pour ça » (Kanor, 2006, p. 143). Il s'agit finalement de restaurer un point de vue fondamentalement humain sur la Déportation transatlantique :

12 «Avant sa publication, Humus a été mis en espace. Deux comédiennes sont montées sur scène pour interpréter les personnages de L'Amazone et de La Muette» (Kanor, 2013, p. 968). 
Mon intention n'était ni de magnifier ni de « gonfler » mes personnages pour en faire des emblèmes, des porte-histoires. [...] J'ai tout simplement voulu conter l'histoire d'Africaines mises en fer, mais qui continuent d'être des femmes, elles vieillissent, elles attendent leurs règles, elles se chamaillent, elles se racontent des histoires, elles se souviennent, elles pensent à leur homme, elles tombent malades, folles ou bien amoureuses. (Kanor, 2013, p. 970)

Les passages qui évoquent leur rapport au corps, à la sexualité et à leur condition de femmes restituent particulièrement les spécificités féminines et intersectionnelles de la Déportation transatlantique, gommées derrière le substantif épicène «esclave ». Ainsi le récit désobéit au regard hégémonique, puisqu'il vise à déconstruire l'essentialisation qui est le propre des oppressions comme celles qui sont liées de la race.

\section{L'hybridité}

\subsection{Dynamiques collectives et identitaires}

Il transgresse également le regard hégémonique en s'appropriant l'oppression comme lieu commun : l'essentialisation est alors reconfigurée en logique fédératrice. Selon l'historien Amzat Boukari-Yabara, c'est en effet l'expérience collective de l'asservissement et de la race qui a impulsé les idéologies politiques à conscience unitaire comme « le panafricanisme et le pan-négrisme - le sentiment d'une unité sur la simple base d'être noir » (Yabara, 2017, p. 9). Dans les œuvres, cette conscience apparaît dans les prémisses de la créolisation, localisée par des penseurs comme Édouard Glissant dans le ventre du bateau négrier, qui enferme ensemble des personnages aux origines ethniques diverses :

Une porte s'est ouverte et je me suis allongée au milieu de ces femmes que je voyais pour la première fois. Baoulés, Yoruba, Ibo, Ashanti, on eût dit que toute l'Afrique sétait donné rendez-vous dans cette geôle, organisée en nations, autour d'une seule et même douleur que je tentais de tromper à force d'histoires. Lorsque je sentis battre les cœurs de nouveau les cœurs, je me décidai à parer pour de vrai. J'avais un plan, il était clair, aussi me levai-je pour l'exposer. (Kanor, 2006, p. 87)

La conscience de la douleur commune et l'union entre les personnages sont ainsi elles aussi reconfigurées en modalités de résistance : le projet du saut, tout comme les histoires d'amour et d'amitié qui se tissent entre elles, constituent des boucliers envers l'anéantissement identitaire : «Ses bras de vieille autour de moi sont des remparts à la haine. Le dégoût de soi »(Kanor, 2006, p. 222). De même, Indigo Mesanedi, qui habite le village localisé en Afrique subsaharienne contemporaine mis en scène dans 
la pièce «Tombeau » (Miano, 2015, pp. 97-173), accueille l'Afro-américaine Jedidiah comme sa « sœur en humanité, [...] en féminité, [...] de mémoire et de douleur, quelle que soit son origine » (Miano, 2015, p.116). Le récit de ces rapports fait écho au principe de sororité, que bell hooks définit à travers la prise en compte de l'hétérogénéité dans des perspectives communes: "Nous pouvons être des sœurs unies par des intérêts et des croyances partagés, unies dans notre appréciation de la diversité, unies dans la lutte que nous menons pour mettre fin à l'oppression sexiste, unies dans la solidarité politique » (hooks, 2008, p. 22). Des logiques similaires sont énoncées par Gilroy à propos de la race : «Le terme 'noir' est certes le produit d'une expérience historique de la violence raciale subie par des groupes humains, mais il désigne également la production utopique d'un commun où les logiques de solidarité naissent de la reconnaissance active des diversités » (Gilroy, 2017, p. 22).

Ces considérations alliant local et global et identité et collectivité se croisent ainsi dans la conscience de l'intersectionnalité des oppressions. Quoique profondément ancrées dans des territoires et des contextes historiques spécifiques ${ }^{13}$, des réseaux de solidarité et d'influences afro-féministes se tissent entre les différents pôles de l'Atlantique noir. Ceux-ci apparaissent aussi dans le cadre de la littérature, à travers un entrecroisement «d'allusions, de références et de choix thématiques se faisant écho les uns les autres »(Burnautzki, 2018, p. 91) ; l'infanticide, mentionné précédemment, en constitue un exemple : circulant au sein de la littérature féminine transatlantique, ce motif était déjà présent dans l'œuvre de l'Afro-américaine Toni Morrison (Beloved, 1987) et de la Guadeloupéenne Maryse Condé (Moi, Tituba, Sorcière... Noire de Salem, 1986), les "aînées " de Kanor et de Miano, qui l'investissent à leur tour ${ }^{14}$ dans leurs textes. Ces réseaux transatlantiques se tissent aussi à travers le croisement, dans l'œuvre des auteures, de personnages féminins qui sont inspirés par des héroïnes historiques ou mythologiques issues des cultures africaines, américaines et européennes. Leur investissement conjoint reflète ainsi une ouverture des frontières, tant des espaces culturels dont ces figures relèvent, que de la perception traditionnelle

13 La situation des femmes noires aux États-Unis differe par exemple fondamentalement de celle des femmes noires en Europe; si la présence des premières est principalement liée à l'histoire de l'esclavage, celle des secondes relève davantage de perspectives postcoloniales. De même, elle obéit à des logiques toutes autres pour les femmes qui vivent sur le continent africain, puisque la race n'y constitue pas l'autorité qu'elle supporte en contexte occidental.

14 «'Comment as-tu osé? L'enfant d'un Blanc c'est comme une chaîne» dit l'Amazone à la Blanche lorsqu'elle apprend qu'elle est enceinte. Elle ajoute «Enfanter ici, c'est donner à manger à l'esclavage » (Kanor, 2006, p. 108). 
des genres : il s'agit de personnalités guerrières, sinon révoltées, et leur corps est investi dans des scènes de confrontation avec le pouvoir en place, détenu majoritairement par des figures masculines :

- «Queen Nanny », figure jamaïcaine du marronnage, à laquelle la pièce « Sacrifices » (Miano, 2015, pp. 53-96) est dédiée, est représentée dans la pièce par le personnage de Maresha. Elle tient une place phare dans la lutte des Marrons, et les personnages masculins lui attribuent de puissantes facultés à combattre : "Maresha est une armée à elle seule. Nous lui devons quelquesunes de nos plus éclatantes victoires. [...] Quand il fallait combattre, c'était son corps entier qu'elle engageait. Jamais la mort ne l'a effrayée » (Miano, 2015, p. 93).

- L’Amazone du roman Humus est inspirée des figures guerrières du royaume de Dahomey. Elle occupait un rôle de meneuse au sein de cette armée féminine : «En ce temps-là, [...] il n'était pas une guerre qui se gagnait sans moi »; "Jamais en queue, devant toujours, brandissant haut le drapeau des artilleurs » (Kanor, 2006, p. 81). « Féministe avant l'heure » (Kanor, 2013, p. 968), elle s'insurge contre la passivité des esclaves et c'est elle qui initie le projet du saut auprès des autres personnages du roman.

- L'histoire de Jedidiah de la pièce "Tombeau " s'apparente au mythe d'Antigone : résidant aux États-Unis, elle se rend au village de ses ancêtres subsahariens qu'elle a localisé grâce à un test $\mathrm{ADN}$, avec le corps de son frère qui souhaitait être enterré dans la terre de ses origines. Soutenue par la prédicatrice Indigo Mesanedi, l'hérö̈ne s'obstinera jusqu'au bout pour que les volontés de son frère soient respectées et ce malgré l'hostilité à laquelle elle se confronte.

En célébrant ensemble ces héroïnes féminines qui ne sont pas toujours reconnues dans les territoires autres que ceux d'où elles proviennent, l'œuvre littéraire des auteures du corpus dessine ainsi les contours d'un lieu d'appartenance transnational en perspective de genres et à l'image de la configuration de l'Atlantique noir. En ce sens, elle peut s'appréhender comme une forme de «tiers-espace », qui vient, ainsi que le définit Homi K. Bhabha «perturber les histoires qui le constituent et établit de nouvelles structures d'autorité, de nouvelles initiatives politiques, qui échappent au sens commun "(Bhabha, 2006, p. 99). Pour les personnages esclaves féminins, cela s'illustre par une appropriation du déplacement forcé et de l'oppression en support de 
mutations identitaires, incluant l'affirmation d'une désappartenance territoriale, laissant ainsi entrevoir une hybridité culturelle assumée :

Tu veux connaître le nom du village. Ne sais-tu pas que je suis de partout ? Je viens des côtes, des grands lacs, des bois, de ces cayes redoutables où les dieux sont moustiques. Je suis née au Dyolof, en Angola, au Loango. Je suis de partout. Partout erre. (Kanor, 2006, p. 217)

Malgré la mention de l'errance, on constate en effet ici que ce n'est pas la négation qui domine, mais bel et bien l'affirmation d'une identité qui englobe des espaces multiples. À partir du vide laissé par la fragmentation s'ouvre finalement de nouvelles possibilités d'être au monde : aussi, le personnage de la Muette se rebaptise « celle-quimarche-le-monde », elle se définit comme celle qui «vient de loin, n'habite plus mais passe » (Kanor, 2006, p. 25). Son parcours s'inscrit dans une dynamique plus globale qui appréhende les traversées au sein de l'Atlantique noir comme profondément inscrites dans l'Afro-descendance, avec pour point de départ le commerce triangulaire, en passant par les mouvements Back to Africa ${ }^{\mathbf{1 5}}$, et allant par ailleurs jusqu'aux migrations contemporaines des Afriques ou des Amériques noires vers l'Europe.

\subsection{Dépassement des genres}

Ces circulations et leur mémoire sont explorées par les œuvres des écrivaines du corpus, qui les investissent aussi comme des possibilités d'ouverture des frontières des genres. Le récit des actes de rébellion et les alternatives à l'ordre oppressif mises en place par des personnages féminins participent du projet de «contre-culture de la modernité ", en ce qu'elles s'opposent à ce que Raewyn Connell nomme la " féminité accentuée », pendant de la «masculinité hégémonique »(Connell, 2014). Ces notions insistent sur la figuration des genres tels qu'ils sont déterminés par la norme sociale, et dans le cas de la féminité, à travers la « conformité au patriarcat » (Connell \& Messerschmidt, 2015, p. 176). Les personnages féminins des auteures se caractérisent donc par des caractères traditionnellement attribués aux hommes, comme le courage, l'initiative etc. (Miano, 2012, p. 31). Le personnage de Maresha par exemple détient des pouvoirs de prédiction, ce qui lui confère un rôle particulier au sein de la communauté. Par ailleurs, à la fin de la pièce, elle défie les habitants des hautes terres et fait figure d'autorité sur la montagne bleue, où elle s'établit. C'est d'elle que

15 Il s'agit de projets de retour initiés par des Afro-américains sur les terres africaines de leurs ancêtres, que l'on retrouve par exemple dans les idéologies du mouvement Rastafari. 
provient la solution au nœud de l'intrigue, puisque les Marrons installés au sein de la nouvelle communauté qu'elle forme continueront d'accueillir des esclaves fugitifs. De nombreux autres personnages féminins de Miano occupent des rôles décisifs : Inyi, " divinité première ", "figure féminine de $\mathrm{Nyambe}^{16}$, médiatrice entre les éléments de la création et porteuse des âmes à naître dans le Premier pays des humains » (Miano, 2015, p. 49) préside le «tribunal » mis en scène dans la pièce « Révélation », et Kalunga, personnage d'apparence « androgyne » (Miano, 2015, p. 15) y occupe le rôle de la Gardienne des passages. De la même manière que Gilroy définit les cultures afro-diasporiques à partir de la frontière atlantique qu'elles ont en partage, Miano définit l'identité sexuelle de ses personnages à partir de «l'entre-deux » (Miano, 2012, p. 31) du féminin et du masculin : c'est dans leur complétude que réside selon elle l'idéal du genre ${ }^{17}$. D'une certaine manière elle en propose une vision queer, celle-ci se définissant comme "l'identité de genre, l'expression de genre, les caractéristiques sexuées ou la sexualité [qui] s'inscrivent passivement ou activement en faux des construits sociaux traditionnels et normatifs » (Martin, 2017, p. 7).

Or, la queer theory est aussi employée pour qualifier des pratiques artistiques à dimension hybride, circulant entre différentes disciplines et dépassant de ce fait les frontières taxinomiques, tout en incluant des arts considérés comme « mineurs ». Il s'agit d'une recherche que Muriel Plana définit comme « sensible aux identités, aux sexualités, mais aussi aux corporéités et à leur articulation avec le social et politique » (Plana, 2016, p. 120) et qui s'intéresse à des esthétiques comme la « scène, plasticité, théâtralité, transgression, subversion, interartisticité, intermédialité, recyclage, etc. » (Plana, 2016, p. 114). Cette approche peut s'appliquer à l'étude des œuvres du corpus, qui tout en remettant en cause les catégories des genres sociaux, se caractérisent par un flottement entre les genres littéraires : si Miano propose dans Red in blue trilogie des textes de théâtre, elle explique qu'en tant que lectrice elle ne fait pas la différence entre poésie, théâtre et roman parce que « la visualisation se fait la même manière » (Miano, 2018). Ainsi, ses textes pour la scène comportent une importante dimension narrative, que soulignent notamment les nombreuses didascalies. Ils comportent aussi des passages à dimension poétique, non ponctués, comme les répliques de la Voix

16 Il s'agit du dieu créateur dans la culture bassa.

17 Dans un article publié aux Inrokuptibles, Miano dit que son «idéal serait d'être homme et femme à la fois». Laffeter, A. \& Sarratia, G. (2017, Mai). Dialogue Virginie Despentes/Léonora Miano. Les Inrockuptibles, 1121, 36-41 (p. 40). https://www.lesinrocks.com/2017/05/23/livres/livres/dialoguevirginie-despentesleonora-miano/ 
du Mort, qui ont été récitées sous forme de slam lors d'une mise en voix présentée au festival d'Avignon ${ }^{18}$.

L'intermédialité permet aussi plus globalement aux auteures de représenter l'hybridité culturelle qui est née de la Déportation transatlantique. Ainsi, entre chaque chapitre de Humus, Kanor insère des chants de marins. La typographie italique employée pour les retranscrire, qui est la même que celle des chants, prières et autres paroles des captives, tisse un réseau transnational au sein du roman :

Au-delà d'une simple chanson de marin, il faut concevoir ce chant comme un requiem, c'est la voix du Nord qui rencontre le Sud, le bruit brutal des hommes qui prennent la mer, la voix du sexe des Blancs qui violent les captives noires, le silence de ces captives face à l'horreur négrière. (Kanor, 2013, p. 973)

Cette rencontre entre dominées et dominants est aussi représentée par l'intermédialité du recueil Red in blue trilogie, qui « renvoie à la mélancolie de la note bleue, à l'origine du blues »(Miano, 2015, p. 3). Naissant dans la violence des plantations, et mêlant les influences africaines à celles de l'Occident, le blues est significatif des cultures «de formation transculturelle et internationale "(Gilroy, 2017, p. 36) que produit l'Atlantique noir. Il illustre des dynamiques de reconfiguration de la douleur en beauté, qui est aussi plus globalement le propre des Afro-descendant·e•s qui héritent du colonialisme :

Car ces identités frontalières sont nées de la douleur. Elles sont nées de l'arrachement, du viol, de la détestation de soi-même. Elles ont dû traverser ces ombres pour inventer un ancrage sur des sables mouvants, et s'imposer, non pas contre, mais parmi les autres. Elles habitent, au fond, un espace cicatriciel. La cicatrice n'est pas la plaie. Elle est la nouvelle ligne de vie qui s'est créée par-dessus. Elle est le champ des possibles les plus insoupçonnés. (Miano, 2012, p. 30)

L'acceptation de la nature frontalière du monde résonne comme une source d'apaisement, ce dont témoigne le cheminement du recueil Red in blue trilogie. Miano appréhende en effet le théâtre à travers sa dimension rituelle. La scène représenterait une place de village où la «famille humaine », femmes et hommes inclus, serait " convoquée », " pour tenter de savoir qui nous sommes, affronter nos ténèbres intérieures » (Miano, 2018). Ainsi, les intrigues sont construites autour d'un conflit à résoudre, ce pour quoi les personnages témoignent tant de leur force que de leur lâcheté,

18 Celle-ci a été diffusée sur RFI Afrique, et le podcast ainsi qu'une interview de Miano à propos de cette lecture sont disponibles sous le lien suivant: http://www.rfi.fr/afrique/20150823-integralleonora-miano-cameroun-red-in-blue-trillogie-tombeau-rfi-theatre 
de leurs ombres - « il s'agit souvent de responsabilités douloureuses, amères » - et de sa complémentarité avec la lumière - « qui se mérite, ne se déploie qu'après qu'on a endossé ses ombres »-, « l'une engendrant toujours l'autre » (Miano, 2012, p. 30). C'est dans cette logique de réconciliation que peuvent aussi s'inscrire des rapports de genre en prise avec d'autres formes d'oppression, pour atteindre finalement des stratégies de soi marquées non plus par la section ou la catégorie, mais au contraire par l'hybridité.

L'hybridité qui surgit de la fragmentation inhérente aux fondements de l'Atlantique noir signifie finalement que « la fin du monde connu n'est pas sa dissolution, mais sa transformation » (Miano, 2016, p. 24), ce qui s'articule aussi au niveau des oppressions intersectionnelles, ainsi que nous avons voulu le montrer au cours de cette étude. Le rapport de domination que le maître impose à l'esclave annihile l'identité de ce dernier, ce qui est particulièrement le cas pour les femmes noires, lesquelles subissent des violences liées à la race se combinant à celles du genre. Toutefois, dans les fractures qu'ont engendrées ces oppressions surgissent des stratégies d'auto-défense : celles-ci s'articulent tant dans le monde des textes, à travers des tactiques de résistance imaginées par les êtres et les corps en situation de domination, que dans celui des lecteur-rice's et des auteures. Fabienne Kanor et Léonora Miano investissent, avec leurs pratiques de création, le terrain laissé vide par l'oblitération des femmes noires au sein de l'histoire, participant de ce fait d'un projet de reconstruction de la mémoire collective qui vise à y inclure les mémoires afro-descendantes. Celles-ci étant fondamentalement marquées par les expériences de circulation, elles performent au sein de leur esthétique le décloisonnement des frontières culturelles. L'Atlantique noir et la littérature qui le raconte ouvre de ce fait des possibilités d'émancipation des différentes formes d'oppressions auxquelles se confronte le sujet noir. Finalement, les marges qui le caractérisent se transforment en « espaces 'interstitiels' [...] qui initient de nouveaux signes d'identité, et des sites innovants de collaboration et de contestation dans l'acte même de définir l'idée de société »(Bhabha, 2007, p. 30).

\section{RÉFÉRENCES BIBLIOGRAPHIQUES}

Aurélia, D. (2012). Voix du Sud :étude de trois autobiographies de femmes esclaves. Transatlantica, 2. http://journals.openedition.org/transatlantica/6229

Bhabha, H. \& Rutherford, J. (2006). Le tiers-espace. Multitudes, 26(3), 95-107. https://www.cairn. info/revue-multitudes-2006-3-page-95.htm

Bhabha, Homi K. (2007 [1994]). Les lieux de la culture: Une théorie postcoloniale (Traduction de Bouillot, Françoise). Payot.

Boukari-Yabara, A. (2014). Africa unite! Une histoire du panafricanisme. La Découverte. 
Burnautzki, S. (2018). Transferts de pratiques militantes et circulations littéraires des critiques black feminist, dans le contexte francophone in G. Bridet, V. Brinker, S. Burnautzki, \& X. Garnier (éds.). Dynamiques actuelles des littératures africaines. Panafricanisme, cosmopolitisme, afropolitanisme (pp. 75-91). Karthala.

Cellier, M. (2018). (Re)cartographies poétiques et imaginaire diasporique : une lecture de l'Atlantique noir à partir d'un Arc-en-ciel pour l'Occident chrétien, Changó el gran putas et The Salt Roads. Études littéraires africaines, 45, 179-197. https://doi.org/10.7202/1051622ar

Chalaye, S. (2020). Race et théâtre. Un impensé politique. Acte Sud-Papier (coll. Apprendre).

Chollet, M. (2018). Sorcières. La puissance invaincue des femmes. La Découverte (coll. Zones).

Cixous, H. (2010 [1975]). Le rire de la Méduse in Le Rire de la Méduse et autres ironies. Galilée.

Connell, R. \& Messerschmidt, J. (2015). Faut-il repenser le concept de masculinité hégémonique? : Traduction coordonnée par Élodie Béthoux et Caroline Vincensini. Terrains E travaux, 27(2), 151-192. https://doi.org/10.3917/tt.027.0151

Connell, R. (2014). Masculinités:enjeux sociaux de l'hégémonie (Traduction de Richard, Claire et al.). Amsterdam.

Crenshaw, K. (2005). Cartographies des marges intersectionnalité, politique de l'identité et violences contre les femmes de couleur. Cabiers du Genre, 39(2), 51-82. https://doi.org/10. 3917/cdge.039.0051

Diop, C. A. (1979 [1954]). Nations nègres et cultures. Présence Africaine.

Dorlin, E. (2005). De l'usage épistémologique et politique des catégories de «sexe» et de «race» dans les études sur le genre. Cabiers du Genre, 39(2), 83-105. https://doi.org/10.3917/cdge. 039.0083

Dorlin, E. (2009 [2006]). La matrice de la race. Généalogie sexuelle et coloniale de la Nation française. La Découverte (coll. Poche).

Dorlin, E. (2017). Se défendre:une philosophie de la violence. La Découverte (coll. Zones).

Etoke, N. (2010). Melancholia Africana. Éditions du Cygne (coll. Mémoires Du Sud).

Herbeck, J. K. (2013). Entretien Avec Fabienne Kanor. The French Review, 5(86), 964-976. https:// core.ac.uk/download/pdf/61745440.pdf

hooks, b. (2008 [1984]). Sororité:la solidarité politique entre les femmes, in Black feminism, Anthologie du féminisme africain-américain, 1975-2000. L'Harmattan, (coll. Bibliothèque du féminisme). https://infokiosques.net/lire.php?id_article=1161

hooks, b. (Traduction de Potot, O.) (2015 [1982]). Ne suis-je pas une femme?: Femmes noires et féminisme. Cambourakis (coll. Sorcières).

Gilroy, P. (2010). L'Atlantique noir. Modernité et double conscience. Amsterdam.

Gontard, M. (1981). La violence du texte. Étude sur la littérature marocaine d'expression française. L'Harmattan.

Kanor, F. (2006). Humus. Gallimard (coll. Continents noirs).

Martin, G. (2017). Chronique linguistique: comment définir le terme identitaire queer? Le Collectif. Québec [consulté le 25/09/2019]. http://www.lecollectif.ca/chronique-linguistiquedefinir-terme-identitaire-queer/

Miano, L. (2012). Habiter la frontière. L'Arche (coll. Tête à tête).

Miano, L. (2015). Red in blue trilogie. L'Arche (coll. Scène Ouverte). 
| HYBRIDA | INTERSECTIONS

Miano, L. (2016). L'impératif transgressif. L'Arche, (coll. Tête à tête).

Miano, L. (2018). Fascicule du spectacle Révélation. Paris: La Colline, Théâtre National. https:// www.colline.fr/spectacles/revelation-red-blue-trilogie

Plana, M. (2017). Pour une approche queer de la littérature et des arts. ElFe XX-XXI Études de littératures de langue française du XX'e et XXI' siècle. A la lumière des études de genre, 6, 113-138. Paris, Classiques Garnier.

Triay, P. (24/01/2014). Fabienne Kanor : "Faire l'aventure“, l'odyssée de l'exil. Outre-mer 1e. Consulté le 26/09/2019 https://la1ere.francetvinfo.fr/2014/01/24/fabienne-kanor-faire-laventure-l-odyssee-de-I-exil-104443.html 